\title{
Influência da forma e do processo de obtenção do entalhe na carga máxima e na energia de fratura de argamassas utilizando o método da cunha para propagação estável de trinca
}

\author{
(Influence of notch shape and preparation on the maximum load and \\ fracture energy of mortars evaluated by the wedge splitting method for \\ stable crack propagation)
}

\author{
S. Ribeiro ${ }^{1}, J . A$. Rodrigues ${ }^{2}$ \\ ${ }^{1}$ Departamento de Engenharia de Materiais - DEMAR, Escola de Engenharia de Lorena - EEL, Universidade de \\ S. Paulo - USP, Lorena, SP 12600-970 \\ ${ }^{2}$ Grupo de Engenharia de Microestrutura de Materiais - GEMM, Departamento de Engenharia de Materiais - \\ DEMa, Universidade Federal de S. Carlos - UFSCar, S. Carlos, SP 13565-905
}

\begin{abstract}
Resumo
Este trabalho mostra a influência da forma e do processo de obtenção do entalhe na estabilidade da propagação de trinca em materiais cerâmicos de microestrutura heterogênea e conseqüentemente, na carga máxima e energia de fratura quando esses materiais são submetidos ao carregamento utilizando o método da cunha. Foram preparadas argamassas com cimento Portland, areia e água, que foram moldadas, curadas a $25{ }^{\circ} \mathrm{C}$ por 7 dias e secadas a $50{ }^{\circ} \mathrm{C}$ por $48 \mathrm{~h}$. Foram estudadas duas proporções (dosagens ou traços) de areia:cimento de 3:1 e 2:1. Foram testados dois tipos de entalhe: um com perfil quadrado da ponta e outro em "V" com ângulo de $60^{\circ}$. Os entalhes de formato quadrado foram obtidos de duas formas: produzidos mecanicamente a partir de disco diamantado e outro na própria moldagem das amostras. Para produzir as amostras entalhadas e ranhuradas já na moldagem, foi desenvolvido um molde de PVC munido de lâminas de aço internas. A partir dos testes preliminares foram estabelecidas as condições de propagação estável da trinca. A trinca percorre um plano imaginário definido pelas ranhuras laterais da amostra. Os resultados mostraram que o entalhe que proporciona melhores condições de estabilidade de propagação de trinca é o de forma em "V", obtido durante o processo de moldagem das amostras.
\end{abstract}

Palavras-chave: método da cunha, propagação estável de trinca, energia de fratura, entalhe, argamassa.

\begin{abstract}
This paper discusses how the shape and preparation of the notch affect crack propagation in ceramic materials with heterogeneous microstructures and, as a result, the maximum load and fracture energy when these materials are subjected to loads using the wedge splitting test. Mortars were prepared with Portland cement, sand and water, which were mixed, molded, and cured at $25{ }^{\circ} \mathrm{C}$ for seven days. After curing, the samples were dried at $55^{\circ} \mathrm{C}$ for $48 \mathrm{~h}$. Two mortar compositions were prepared with proportions of sand:cement of 3:1 and 2:1. Two types of notches were tested: one with a square-tipped profile and the other V-shaped with a $60^{\circ}$ angle. The square shaped notches were prepared in two ways: one mechanically with a diamond disc tool and the other using blades as cores during the molding of the mortars. A PVC mold equipped with internal steel blades was created to produce samples notched and grooved in the mortar molding process. Stable conditions of crack propagation were established based on preliminary tests. The crack was found to propagate along an imaginary plane defined by the lateral grooves in the sample. The results indicated that the notch providing the best conditions of crack propagation stability is the V-shaped notch produced in the molding of the sample.
\end{abstract}

Keywords: wedge splitting method, stable crack propagation, fracture energy, notch, mortar.

\section{INTRODUÇÃO}

Concreto é um material heterogêneo formado por uma estrutura dura de cimento e partículas de rocha, os agregados, que exibe comportamento quase-frágil. É o material mais comumente usado na construção civil no mundo inteiro, daí a necessidade crescente do entendimento do seu comportamento mecânico, das propriedades de seus componentes e, particularmente, o trincamento e comportamento de fratura [1-5].

A produção de um concreto se inicia com a mistura da areia com cimento, agregado (normalmente as britas) e água em proporções adequadas. Após a mistura, o concreto é curado. Essa fase é muito importante, pois nela ocorrem as reações de hidratação e, conseqüentemente, as ligações químicas que lhes conferem resistência mecânica. Sabe-se que o tempo de cura 
quase completa de um concreto é de 28 dias, porém com sete dias o concreto já adquire $80 \%$ de sua resistência total $[4,6,7]$. Um fenômeno muito interessante e bastante estudado, em outras áreas, é o efeito de parede observado quando da moldagem de argamassas e concretos. Esse efeito está relacionado à afinidade das partículas finas pelas paredes do recipiente que contém a argamassa ou o concreto em relação às partículas mais grossas (agregados) $[8,9]$.

Sob o ponto de vista da engenharia, a resistência mecânica é a mais importante propriedade do concreto estrutural, porém a energia de fratura, $\gamma_{\text {wof }}$, é relevante para o projeto de grandes estruturas de concreto porque é uma propriedade que avalia a resistência à propagação de trincas $[10,11]$. A energia de fratura pode ser definida como o trabalho médio por unidade de área projetada de fratura para propagar uma trinca, representada pela soma de energias associadas a diferentes mecanismos de absorção ou dissipação durante o processo de fratura. O seu valor pode ser determinado a partir da curva carga-deslocamento gerada por um ensaio de propagação estável de trinca e da seguinte equação [12-14].

$$
\gamma_{\text {wof }}=\frac{1}{2 \mathrm{~A}} \int \mathrm{P}_{\mathrm{M}} \mathrm{d} \delta
$$

em que A é a área projetada da superfície de fratura, $\mathrm{P}_{\mathrm{M}}$ é a carga vertical aplicada pela máquina de ensaios e $\delta$ é $\mathrm{o}$ deslocamento do atuador da máquina. $\mathrm{O}$ valor da integral $\int \mathrm{P}_{\mathrm{M}} \mathrm{d} \delta$ é determinado pela área sob a correspondente curva carga-deslocamento.

Para se avaliar experimentalmente a energia de fratura, a condição essencial é que a propagação da trinca seja estável e se tratando de argamassas e concretos em que possa se trabalhar com agregados de vários tamanhos, as dimensões das amostras é uma preocupação importante. Trabalhando com amostras pequenas, a seção a ser fraturada pode conter agregados com tamanhos relativos próximos ao dessa seção, produzindo resultados insatisfatórios. A desvantagem de se utilizar grandes amostras está no acúmulo de energia elástica e quando da propagação da trinca essa se faz de modo instável [15-18].

Para avaliar a energia de fratura, a condição de propagação estável da trinca deve ser rigorosamente obedecida e para isso, a energia acumulada na amostra e na máquina de ensaios durante a deformação elástica deve ser a menor possível e transformada em energia de superfície de modo controlado, sendo que a condição necessária para tal pode ser descrita pela seguinte equação:

$$
\frac{\mathrm{d}\left(\mathrm{U}_{\mathrm{M}}+\mathrm{U}_{\mathrm{S}}\right)}{\mathrm{dc}} \leq \frac{\mathrm{dW}_{\mathrm{fs}}}{\mathrm{dc}}
$$

em que $\frac{d\left(U_{M}+U_{S}\right)}{d c}$ é a variação (no sentido de liberação) das energias elásticas armazenadas na máquina $\left(U_{M}\right)$, incluindo assessórios e na amostra $\left(U_{S}\right)$ por unidade de propagação de trinca $(d c)$ e $\frac{\mathrm{dW}_{\mathrm{fs}}}{\mathrm{dc}}$ é a variação da energia de superfície $\left(d W_{f s}\right)$ consumida por unidade de propagação de trinca [15, $16,18]$.

A estabilidade da propagação de uma trinca pode ser avaliada a partir da curva carga-deslocamento gerada durante o ensaio. A Fig. 1 ilustra uma curva carga-deslocamento completa e uma ampliação da região imediatamente antes e após a sua carga máxima. Essa região é crítica, pois pode ocorrer uma instabilidade na propagação da trinca e a mesma expandir-se catastroficamente.

Para trabalhar com amostras relativamente grandes e
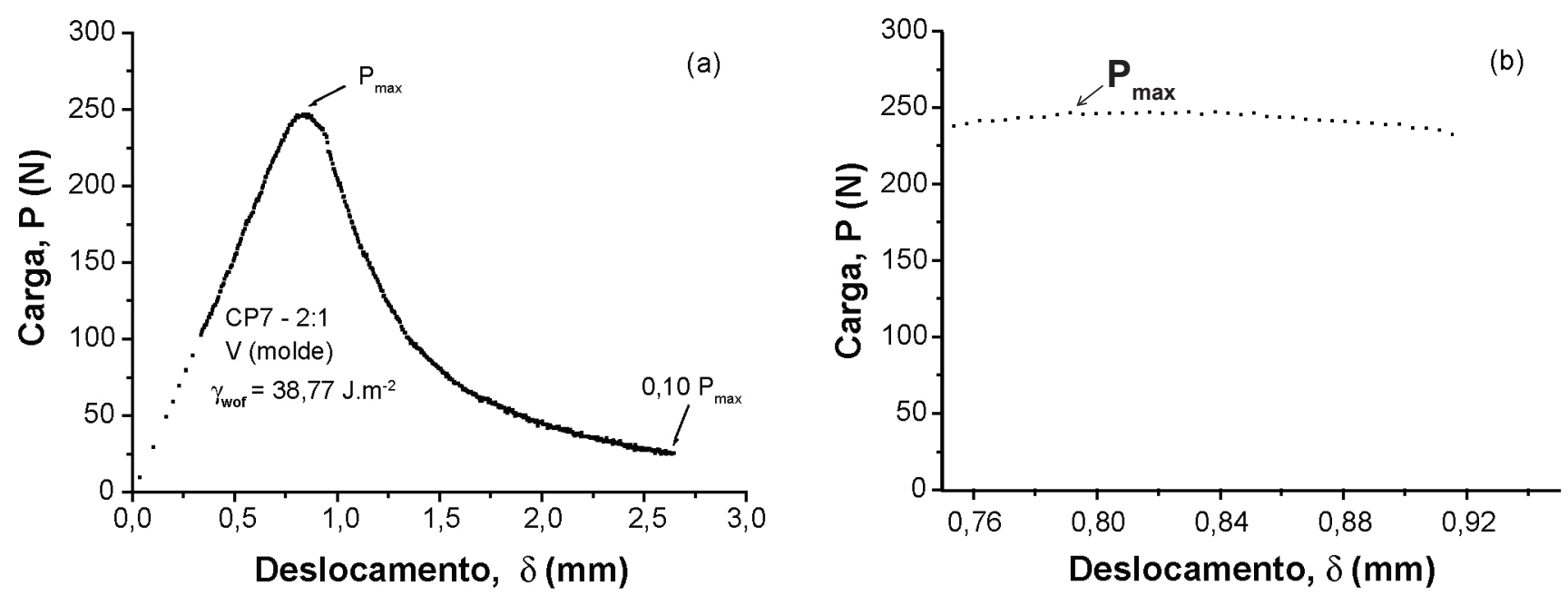

Figura 1: Curva carga-deslocamento obtida para uma das amostras testadas: (a) curva típica de propagação estável de trinca e (b) ampliação da região próxima da carga máxima mostrando o comportamento bastante estável antes e após esse ponto que é considerado crítico.

[Figure 1: Load-displacement curve from a sample tested in this work: (a) typical stable crack propagation curve, and (b) magnification of the critical region close to the maximum load, showing the stable behavior before and after that point.] 


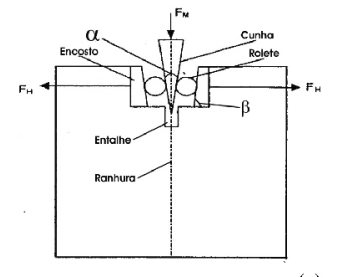

(a)
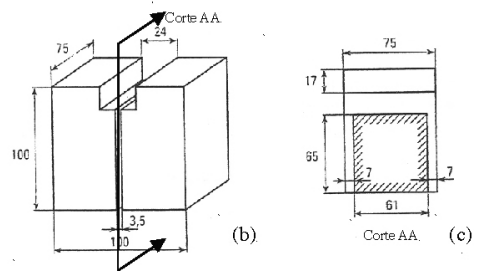

Figura 2: (a) Arranjo esquemático do método da cunha, em que $\alpha$ e $\beta$ são, respectivamente, os ângulo da cunha e dos encostos [21,23]. (b) geometria e dimensões da amostra (em mm) e (c) corte "AA" da secção transversal do corpo de prova (parte b) passando pelo entalhe e ranhuras laterais [23].

[Figure 2: (a) Schematic diagram of the wedge splitting method, where $\alpha$ e $\beta$ are wedge and support angles, respectively [21, 23]. (b) geometry and dimensions of the sample $(\mathrm{mm})$, and (c) "AA" crosssection of $(b)$ passing through notch and lateral grooves [23].]

sem o problema do armazenamento de grandes quantidades de energia na amostra e na máquina, Tschegg patenteou um método chamado "Wedge Splitting Test" [19], que é o método da abertura da trinca empregando uma cunha, ou simplesmente "Método da Cunha". Depois disso, vários outros pesquisadores vêm contribuindo para implantação e melhoria do método que é aplicado não só para concretos da construção civil como também para concretos refratários [3, 5, 20-30].

Como pode ser visto na Fig. 2a, a cunha recebe a carga da máquina, $\mathrm{F}_{\mathrm{M}}$, e a transmite para os roletes e estes para os encostos que produzem as forças horizontais, $\mathrm{F}_{\mathrm{H}}$, na amostra sob teste. A Fig. $2 \mathrm{~b}$ apresenta a geometria da amostra e suas dimensões típicas [21, 23] e na Fig. 2c vêem-se os detalhes do entalhe, das ranhuras e da secção de fratura após o teste.

A principal vantagem do método da cunha baseia-se na possibilidade de se trabalhar com amostras relativamente grandes, para as quais o tamanho do agregado torna-se suficientemente pequeno para resultar em uma relação agregado/dimensões transversais pequena. Ainda nesse método, a força aplicada pela máquina de ensaio é bastante reduzida devido ao efeito da cunha, conforme a seguinte equação $[21,23,30]$ :

$$
F_{H}=\frac{F_{M}}{2 \tan \left(\frac{\alpha}{2}\right)}
$$

em que $\alpha$ é o ângulo da cunha, $F_{H}$ é a força horizontal produzida a partir de $F_{M}$, que é a força vertical aplicada pela máquina de ensaios. No caso de se utilizar $\alpha$ igual a $10^{\circ}, F_{H}$ é igual a $5,71 \times F_{M}$.

Além de se utilizar a cunha para diminuir a carga necessária aplicada pela máquina para a abertura da trinca, pode-se também utilizar entalhes com ângulos de curvatura pequenos, pois esses são concentradores de tensão e favorecem a abertura da trinca com menores cargas iniciais, ajudando na estabilidade da propagação da trinca, principalmente nos instantes da transição do regime elástico para o início do avanço da trinca. A concentração de tensão na ponta de uma trinca em função do raio de curvatura pode ser descrita pela equação proposta por Inglis [31]

$$
\sigma_{c}=\sigma_{a}\left[1+2\left(\frac{\mathrm{c}}{\rho}\right)^{\frac{1}{2}}\right]
$$

em que $\sigma_{c}$ e $\sigma_{a}$ são as tensões na ponta da trinca e aplicada, respectivamente, c é o tamanho da trinca e $\rho$ é o raio de curvatura da ponta da trinca.

Vários trabalhos foram desenvolvidos avaliando o tamanho e o raio de curvatura da ponta do entalhe iniciador da trinca nas medidas de tenacidade à fratura, curvas $\mathrm{R} \mathrm{e} \mathrm{J}$, propagação estável de trinca, entre outros [32-36].

No método da cunha, as amostras possuem entalhe e ranhuras laterais coplanares (vide Fig. 2). As ranhuras definem o plano de propagação da trinca, evitando que a mesma se desvie para outras regiões da amostra $[21,23,26-$ $28,37,38]$.

O principal objetivo deste trabalho foi determinar o melhor processo de obtenção e a forma do entalhe para preparação de corpos de prova usados no método da cunha, avaliando-se a carga máxima, a energia de fratura e a estabilidade das curvas carga-deslocamento, visando ampliar as possibilidades de melhor caracterizar materiais cerâmicos de microestrutura heterogênea e grosseira tais como argamassas e concretos.

\section{MATERIAIS E MÉTODO}

Foi desenvolvido um molde especial com lâminas de aço internas para produzir, durante a moldagem das amostras, as ranhuras e o entalhe. O molde original de PVC foi desenvolvido anteriormente em trabalho relacionado ao método da cunha, para avaliação da energia de fratura de materiais cerâmicos tipo argamassas e concretos [17]. A Fig. 3 mostra uma fotografia do molde montado. Este molde tem suas dimensões adequadas para a elaboração de amostras medindo $100 \times 100 \times 75 \mathrm{~mm}^{3}$.

Para obtenção das amostras foram usados: areia média lavada para construção civil, com tamanho médio de partículas de $500 \mu \mathrm{m}$ e tamanhos máximo e mínimo de 2800 $\mu \mathrm{m}$ e $53 \mu \mathrm{m}$, respectivamente; cimento Portland CPII-Z-32, Companhia de Cimento Portland Itaú, fabricado conforme norma brasileira NBR 11578/1991 e água potável.

Foram utilizadas duas composições mássicas em termos de areia:cimento de $2: 1$ e 3:1. A quantidade de água teve como base de cálculo 0,46 da massa de cimento. A areia e o cimento foram pesados e misturados previamente. Após isso, a água foi adicionada aos poucos com o sistema de mistura em movimento, permitindo uma perfeita interação entre os três constituintes da argamassa.

A argamassa foi despejada no molde de PVC, sob a ação de uma mesa vibratória que auxilia a sua acomodação no interior do molde e também ajuda na expulsão de bolhas de ar. As amostras foram curadas por sete dias em atmosfera 


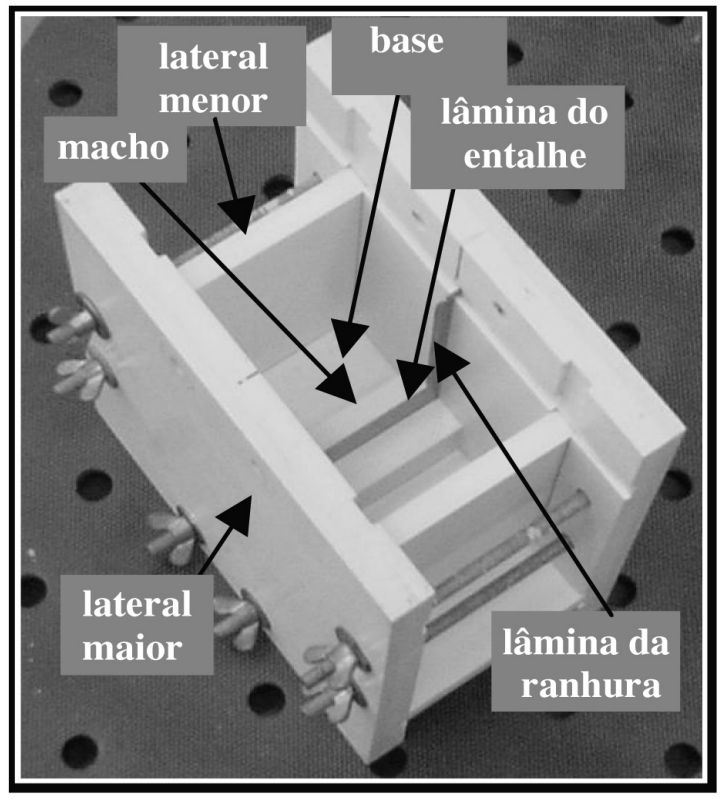

Figura 3: Molde confeccionado em PVC rígido munido de lâminas de aço para produzir as ranhuras laterais e entalhe nas amostras moldadas.

[Figure 3: Rigid PVC mold equipped with steel blades to form the notch and lateral grooves in molded samples.]

saturada de água a $25^{\circ} \mathrm{C}$ e secadas a $50{ }^{\circ} \mathrm{C}$ por $48 \mathrm{~h}$.

Para se verificar o efeito do formato e do processo de obtenção do entalhe, parte das amostras foi preparada no molde mostrado na Fig. 3 sem as lâminas de aço e posteriormente entalhada e ranhurada utilizando-se um disco diamantado com perfil quadrado. A outra parte de amostras foi entalhada e ranhurada durante a própria moldagem, empregando-se lâminas de aço específicas para produzir perfis quadrado e em " $\mathrm{V}$ " com ângulo de aproximadamente $60^{\circ}$ para a ponta dos entalhes. O entalhe e as ranhuras mediam 1,2 $\mathrm{mm}$ de espessura e suas profundidades eram de 15 e $7 \mathrm{~mm}$, respectivamente.

As amostras foram retificadas nas suas bases com o objetivo de se manter o alinhamento com o eixo da máquina de ensaio. Esta condição é obrigatória para que as forças horizontais estejam balanceadas entre os dois lados do entalhe.

Para o teste de propagação estável de trinca, foi utilizada uma máquina de ensaios mecânicos, servo-hidráulica, da marca MTS, série 810, com célula de carga de 50 kN, com características de "closed loop", controle TestStar IIs. Os testes foram controlados pelo deslocamento do atuador, com velocidade de $20 \mu \mathrm{m} \cdot \mathrm{min}^{-1}$. Essas condições foram fixadas a partir de testes preliminares.

Com os dados de deslocamento do atuador e da força instantâneos, foram elaboradas as curvas cargadeslocamento. A partir dessas curvas, foi determinado o trabalho de fratura, extraindo-se a área sob as mesmas e dividindo-as pela área de fratura projetada multiplicada por dois, de acordo com a equação (A), encontrando-se, assim, $\gamma_{\text {wof }}$. A integral sob a curva foi feita até o ponto em que a carga caia ao valor de $10 \%$ da carga máxima, $\mathrm{P}_{\text {máx }}$, atingida no teste. Vide Fig. 1a. Para cada condição de amostra (entalhe e composição), foram determinadas no mínimo, 5 curvas carga-deslocamento, das quais se obtiveram os valores médios de $\gamma_{\text {wof }}$ e $\mathrm{P}_{\text {máx. }}$.

A Fig. 4a ilustra um corpo de prova produzido no molde de PVC com seus detalhes e na sua parte (b), um corpo de prova com o sistema da cunha montado e pronto para execução do teste de propagação de trinca pelo método da cunha. O rebaixo é obtido na própria moldagem e deve ser rigorosamente alinhado, pois neste local são assentados os encostos, que recebem os roletes e a cunha.
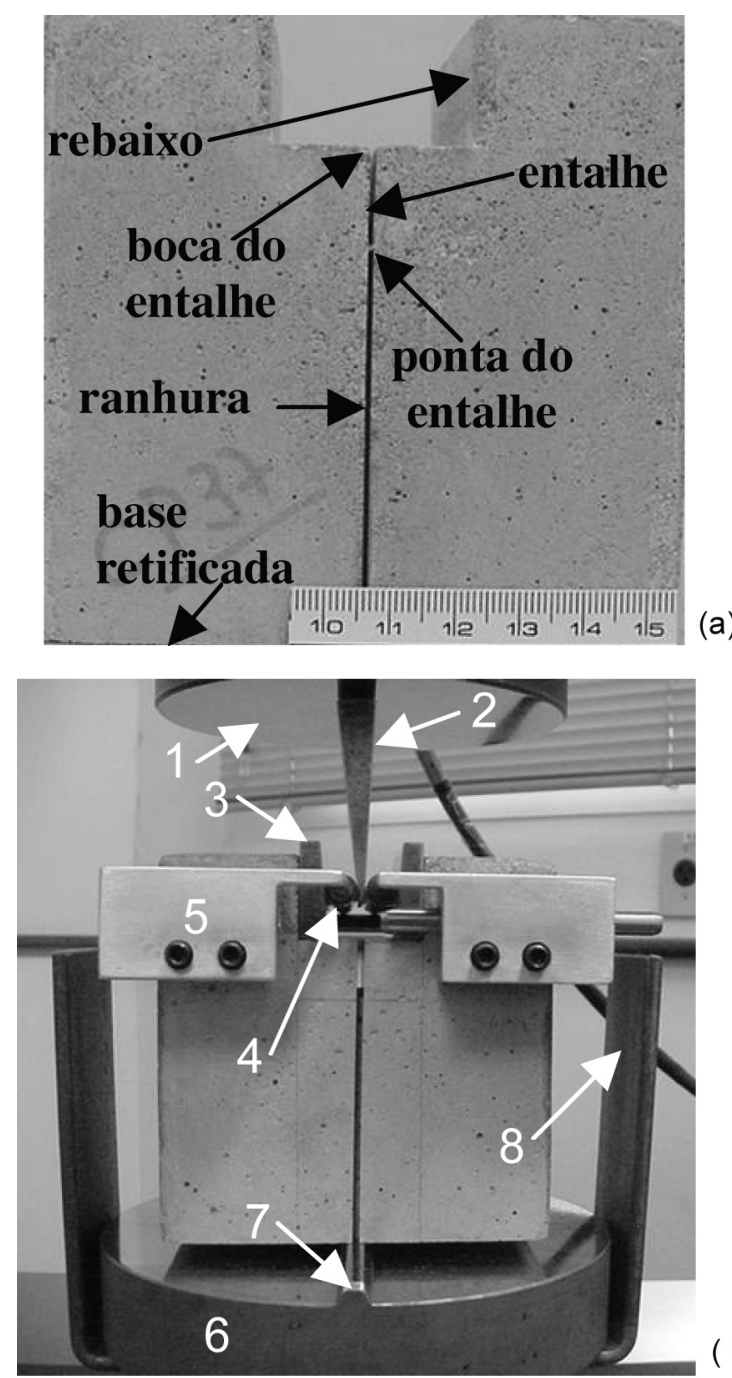

( b )

Figura 4: (a) Corpo de prova moldado em molde de PVC mostrando as suas principais partes: rebaixo, entalhe e ranhura, (b) Corpo de prova com o dispositivo da cunha montado: 1 - placa superior acoplada à célula de carga da máquina, 2 - cunha, 3 - encosto, 4 rolete, 5 - dispositivo auxiliar da montagem do sistema da cunha, 6 - placa inferior acoplada ao atuador da máquina, 7 - apoio do corpo de prova e 8 - dispositivo de segurança.

[Figure 4: (a) Molded sample produced in the PVC mold, showing its principal parts: rabbet, notch and groove; (b) Sample with mounted wedge device: 1 - upper plate connected to the load cell, 2 -wedge, 3 -support, 4 - roller , 5 - auxiliary wedge system mounting device, 6 - lower plate connected to the actuator, 7 sample support, and 8 - safety device.] 


\section{RESULTADOS}

Na Fig. 5 estão mostrados os entalhes (a) produzido mecanicamente com o disco diamantado de perfil quadrado, (b) produzido na moldagem, de perfil quadrado e (c) produzido na moldagem, de perfil em "V". Observando-se os perfis quadrados percebe-se que aquele obtido na moldagem tem os cantos mais agudos do que aquele produzido com o disco. O motivo dessa diferença é que o próprio disco, por razões de fabricação ou até mesmo por desgaste, o seu perfil não é de cantos vivos. Também, o disco utilizado produziu um entalhe ligeiramente mais largo que os da moldagem, ou

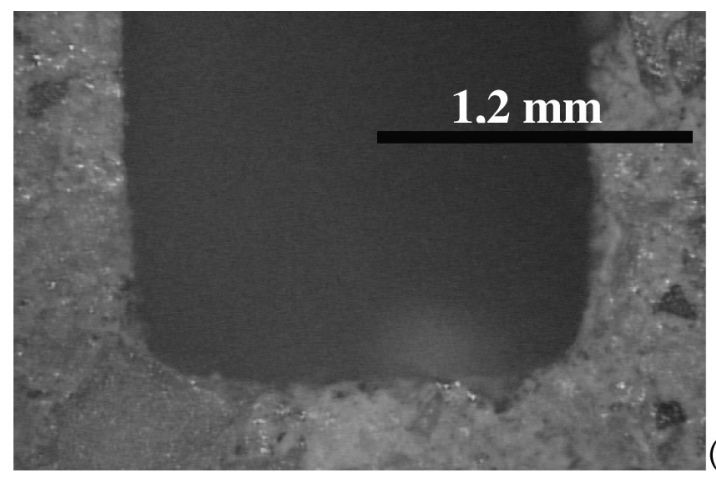

(a)

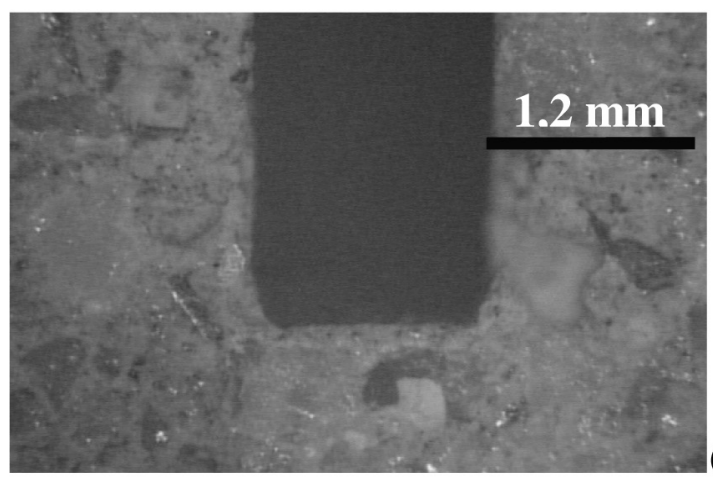

(b)

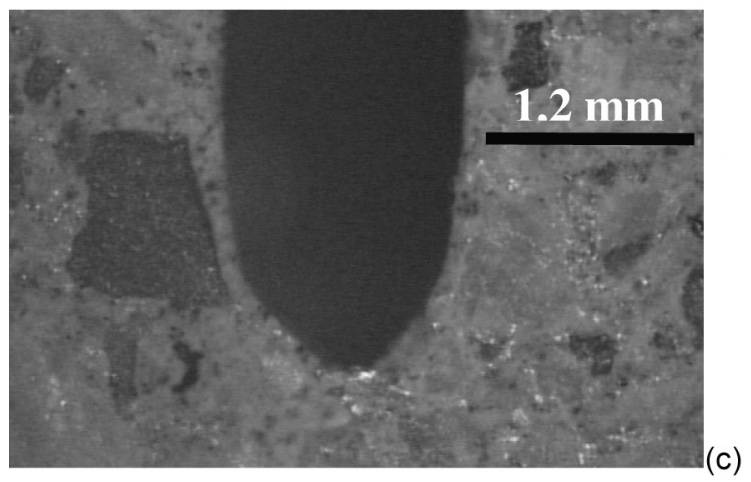

Figura 5: Fotografias mostrando os perfis dos entalhes dos corpos de prova usados nos testes de propagação estável de trinca pelo método da cunha: (a) quadrado obtido mecanicamente com disco diamantado, (b) quadrado obtido na moldagem e (c) em "V" obtido na moldagem.

[Figure 5: Photographs of the profile of the notches in the samples used in stable crack propagation tests by the wedge splitting method: (a) square profile produced with the diamond disc tool, (b) square profile produced during molding, and (c) V-shaped profile produced during molding.] seja, 1,7 mm contra 1,2 $\mathrm{mm}$.

Para uma avaliação mais detalhada da parede do entalhe, mais precisamente no seu canto, foram feitas observações utilizando microscopia óptica, cujas fotomicrografias podem ser vistas na Fig. 6. Na Fig. 6a nota-se que a parede do entalhe é bastante lisa e que o agregado foi cortado pelo disco diamantado (ver as setas indicadoras). Essa característica é observada mesmo na região inter-agregados formada pela matriz. Já na Fig. 6b, vê-se claramente a formação de uma camada constituída aparentemente por um material mais fino, nesse caso pela matriz de cimento. Isso é natural, pois quando argamassas ou concretos são moldados ocorre o fenômeno conhecido como efeito de parede em que as partículas mais finas se dirigem preferencialmente para as paredes das caixas de moldagem e para as superfícies das partículas maiores.

A Fig. 7 mostra fotografias de duas amostras fraturadas dando destaque à região imediatamente vizinha à frente
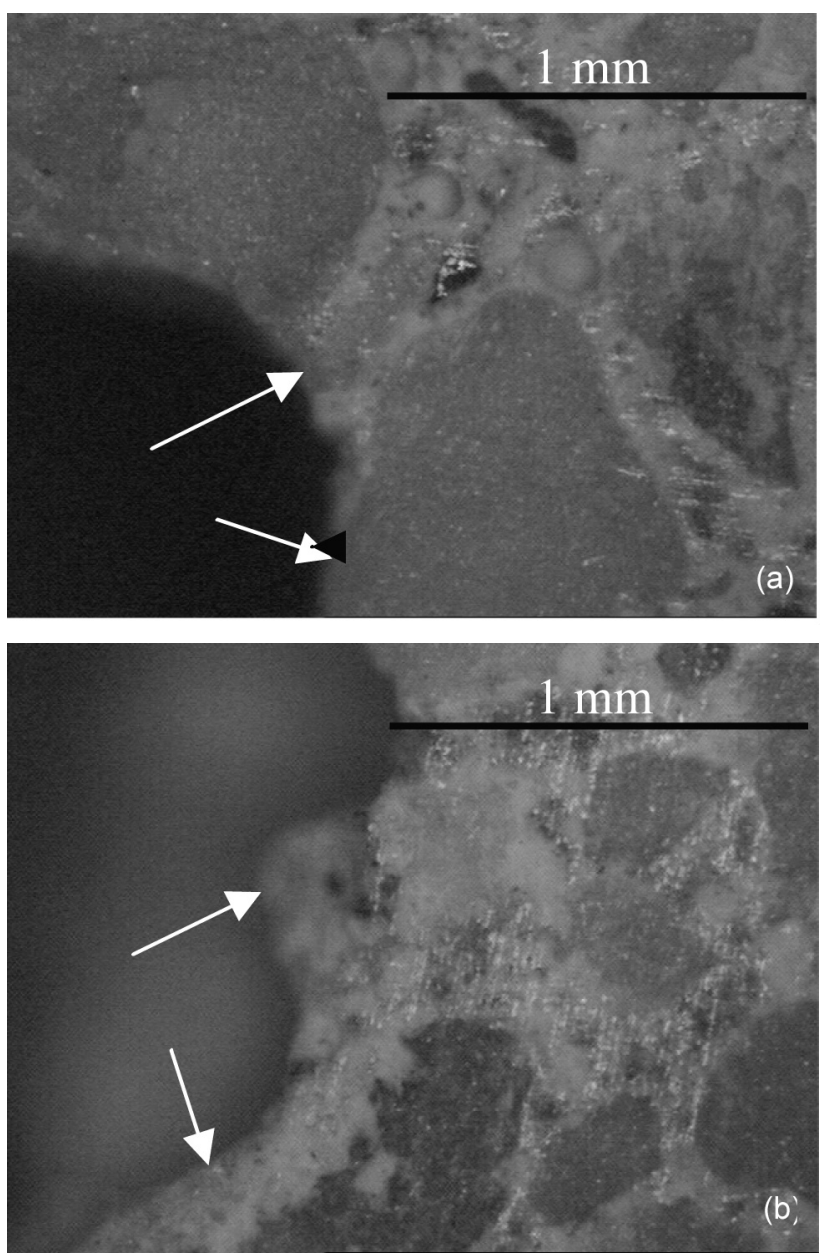

Figura 6: Fotomicrografias obtidas por microscopia óptica mostrando detalhes da região da ponta do entalhe das amostras: (a) entalhada mecanicamente com disco de corte e (b) entalhada durante a moldagem.

[Figure 6: Photomicrographs obtained by optical microscopy showing details of region at the tip of the notch: (a) notch produced mechanically with the diamond disc cutting tool, and (b) notch formed during molding.] 


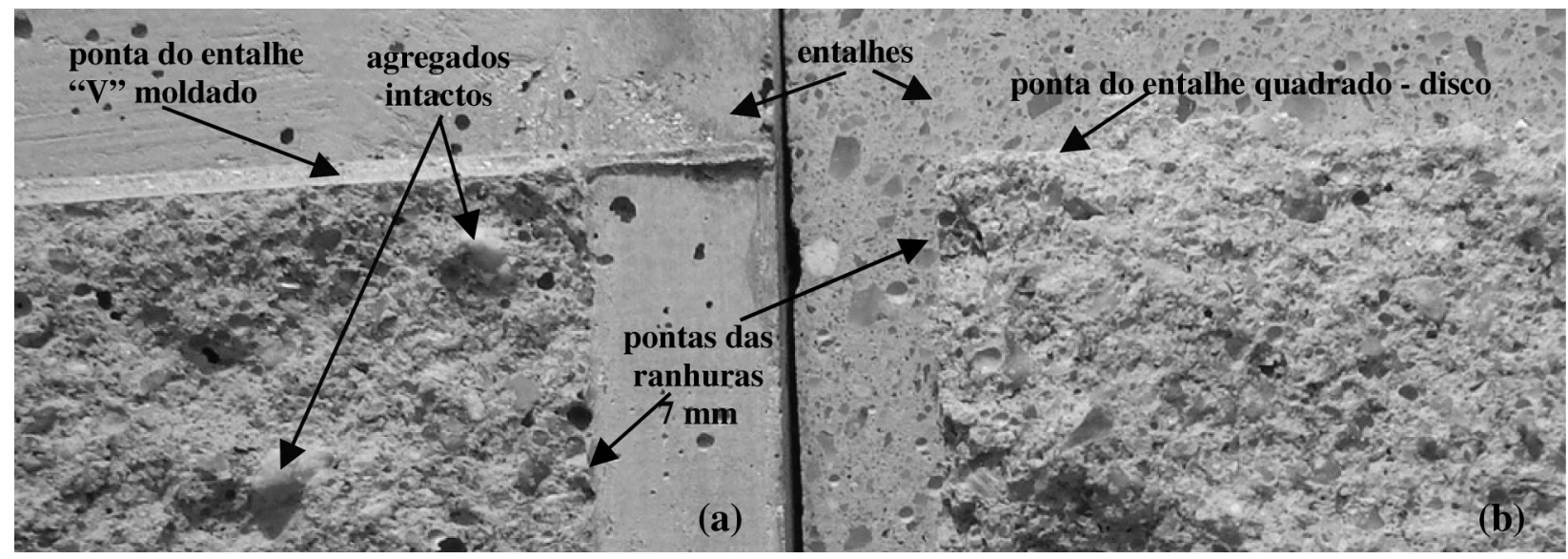

Figura 7: Fotografias das amostras fraturadas da argamassa 2:1: (a) entalhada na moldagem, ponta com perfil em "V" e (b) entalhada com disco diamantado, ponta com perfil quadrado.

[Figure 7: Photographs of the fractured mortar sample with 2:1 composition: (a) notched in the mold, V-shaped profile, and (b) sample notched with diamond disc tool, square-shaped tip.]

do entalhe e das ranhuras. Nota-se claramente a diferença entre as superfícies da parede do entalhe e ranhuras quando preparados na moldagem, parte (a) da Fig. 7, ou com disco diamantado, parte (b) da mesma figura. A superfície do entalhe e das ranhuras obtidos na moldagem é constituída de partículas muito finas da matriz devido ao efeito de parede, enquanto na superfície produzida por ação mecânica de um disco é constituída de agregados cortados e matriz de igual composição granulométrica de todo o volume da amostra. Também, na Fig. 7b, região da ranhura e entalhe, pode-se ver a estrutura da argamassa, constituída por agregados (parte grossa) e pela matriz (parte fina).

Outra observação importante que pode ser feita é o comportamento da interface agregado/matriz onde os agregados são separados da matriz por destacamento, pois os mesmos aparentam estar intactos, sem fraturamento, indicando que a energia necessária para sua fratura é maior que a necessária para destacá-los da matriz.

A Fig. 8 mostra seis curvas representativas de todas obtidas neste trabalho. Com isso, é possível ilustrar a questão da estabilidade de propagação da trinca durante o teste. Uma análise geral da Fig. 8 mostra que as amostras entalhadas mecanicamente, com perfil quadrado, curvas (a) e (d), apresentam situações de curta instabilidade bem mais significativas que as entalhadas na moldagem com o mesmo perfil, curvas (b) e (e), para ambas as composições. Isto pode ser justificado pelas cargas máximas exigidas para a propagação das trincas, pois as amostras entalhadas mecanicamente atingiram valores médios da ordem de 270 e $376 \mathrm{~N}$, para $3: 1$ e 2:1, respectivamente, enquanto as entalhadas na moldagem 214 e 288 , para $3: 1$ e 2:1, respectivamente. Sabe-se que quanto maior a carga máxima, mais energia elástica é armazenada na máquina e na amostra, maior a probabilidade de uma liberação rápida dessa energia e, consequentemente, instabilidade na propagação. Ainda, analisando-se a Fig. 8, curvas (c) e (f) e comparado-as com as demais, (a) e (d) e (b) e (e) verifica-se que as mesmas têm comportamentos mais estáveis que todas. Nesse caso, pode-se também justificar pelas menores cargas máximas alcançadas durante a propagação. As cargas máximas exigidas foram 182 e $228 \mathrm{~N}$, respectivamente, representadas pelas curvas (c) e (f). A justificativa para os níveis mais baixos de carga máxima para as amostras entalhadas na moldagem e com perfil em "V" está fundamentada no mecanismo de concentração de tensão na ponta da trinca (entalhe) estudado amplamente na mecânica da fratura e que pode ser avaliado pela equação (D).

Os gráficos das Figs. 9 e 10 mostram, respectivamente, os resultados de carga máxima e energia de fratura das amostras de composições 2:1 e 3:1, entalhadas e ranhuradas mecanicamente e durante a moldagem. Nesses gráficos estão também indicadas as formas de entalhe utilizadas, ou seja, perfiz quadrado e em "V". Observa-se na Fig. 9 que existe uma diferença significativa nas cargas máximas alcançadas durante a propagação da trinca quando se utilizam formas e maneiras diferentes de produzir o entalhe iniciador da trinca, para ambas as composições de argamassa. A diferença mais acentuada se observa para a composição 2:1. A explicação para maiores diferenças provavelmente está na maior rigidez e maior resistência mecânica das amostras de composição 2:1 devido a maior quantidade de cimento utilizada e, portanto, maior coesão do sistema. A justificativa para um maior valor de carga máxima para amostras entalhadas mecanicamente em relação ao mesmo perfil, quadrado, obtido na moldagem, pode estar associado, primeiramente, ao fato de que o entalhe feito mecanicamente acabou saindo mais largo e mais "arredondado". Isso equivaleria a um entalhe com raio de curvatura da ponta maior, o que causaria menor concentração de tensão, de acordo com a equação (D) e, por isso, exigindo maior carga máxima no ensaio. Outra possibilidade seria, também, o efeito das partículas dispostas na região exatamente na ponta do entalhe iniciador da trinca. Ao longo da frente do entalhe obtido pela ação do disco diamantado, tanto a matriz quanto os agregados estão dispostos de maneira "uniforme", pois foi retirado material suficiente para atingir uma região sem "defeitos" 

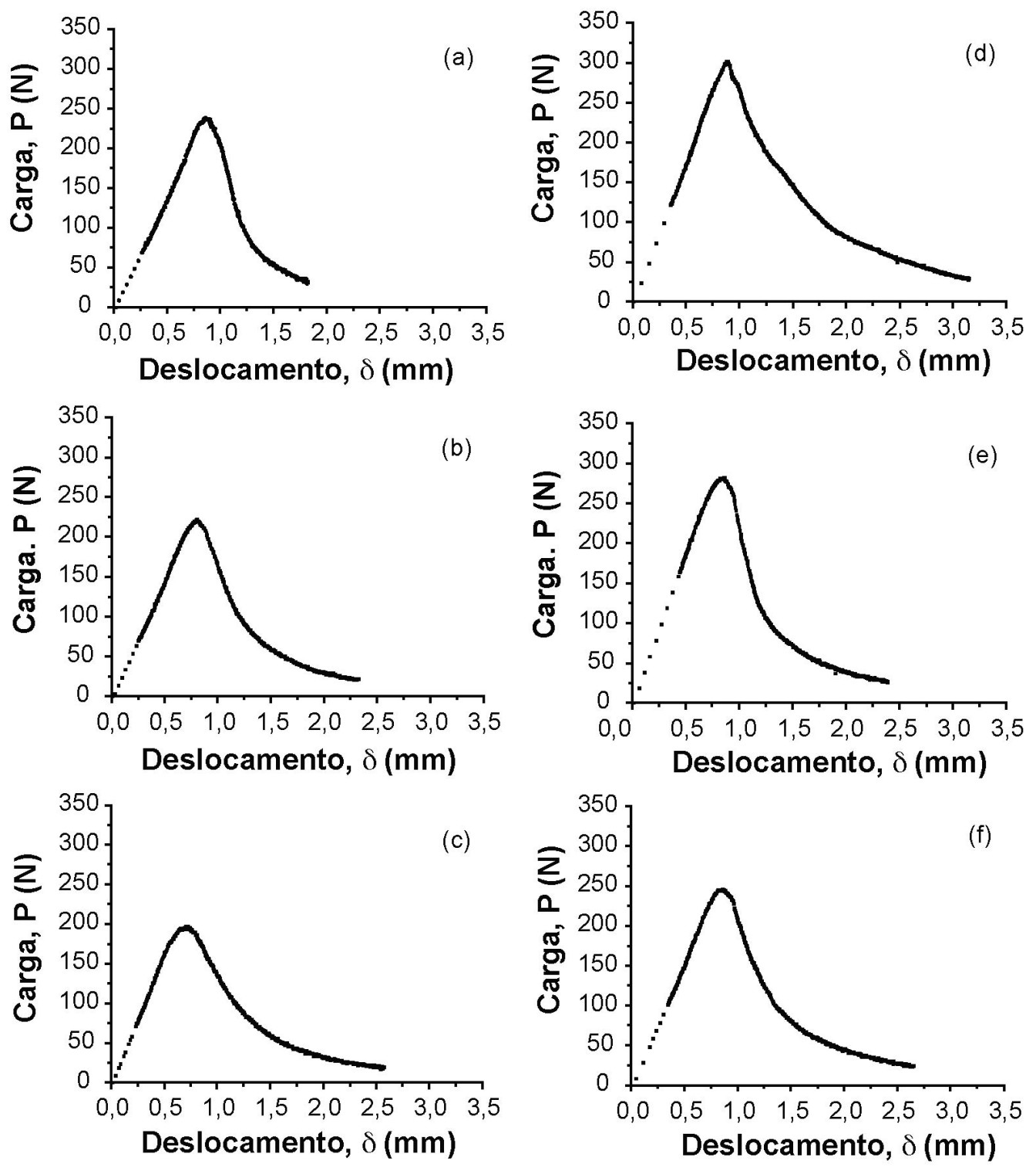

Figura 8: Curvas carga-deslocamento de amostras escolhidas para representar um lote usado na determinação da energia de fratura das argamassas de composições 3:1 (a-c) e 2:1 (d-f) utilizando diferentes modos de confecção do entalhe e ranhuras: (a e d) mecanicamente com disco diamantado com perfil quadrado, (b e e) na moldagem com lâminas com perfil quadrado e (c e f) na moldagem com lâminas com perfil em "V" de $60^{\circ}$.

[Figure 8: Load-displacement curves of samples representing a lot used for the determination of the fracture energy of mortars with compositions 3:1 (a-c) and 2:1 (d-f), with notches and grooves formed in different ways: (a and d) mechanically, with the diamond disc tool with square profile, ( $b$ and e) formed during molding with square profile, and ( $c$ and f) formed in the mold with V-shaped profile.]

de superfície de moldagem. Já na mesma região, para uma frente do entalhe moldado a superfície se encontra com uma distribuição de partículas de forma bastante aleatória, inclusive afetada pelo fenômeno do efeito de parede, que gera um acúmulo de partículas mais finas em relação ao restante do corpo da amostra (ver Fig. 6). Nessa região, é provável que haja a geração de microtrincas e até mesmo trincas, que poderão contribuir para a diminuição da carga necessária para iniciar a propagação. A diferença da microestrutura nas superfícies das paredes do entalhe nas amostras entalhadas mecanicamente e na moldagem já foi vista na Fig. 6. Podese observar que a ponta do entalhe obtido mecanicamente tem uma superfície lisa e mais uniforme promovida pela ação do disco enquanto na superfície da ponta do entalhe moldado verifica-se uma superfície "irregular" e constituída por uma camada de partículas finas e somente após certa distância percebe-se a presença de partículas maiores de agregado. Portanto, existe uma região em que a constituição microestrutural é diferente do interior do volume da amostra.

Avaliando-se a Fig. 10, nota-se que a energia de fratura segue a mesma tendência que a carga máxima, observada na Fig. 9, ou seja, a energia de fratura é maior para as amostras que apresentaram a maior carga máxima. Isso está 

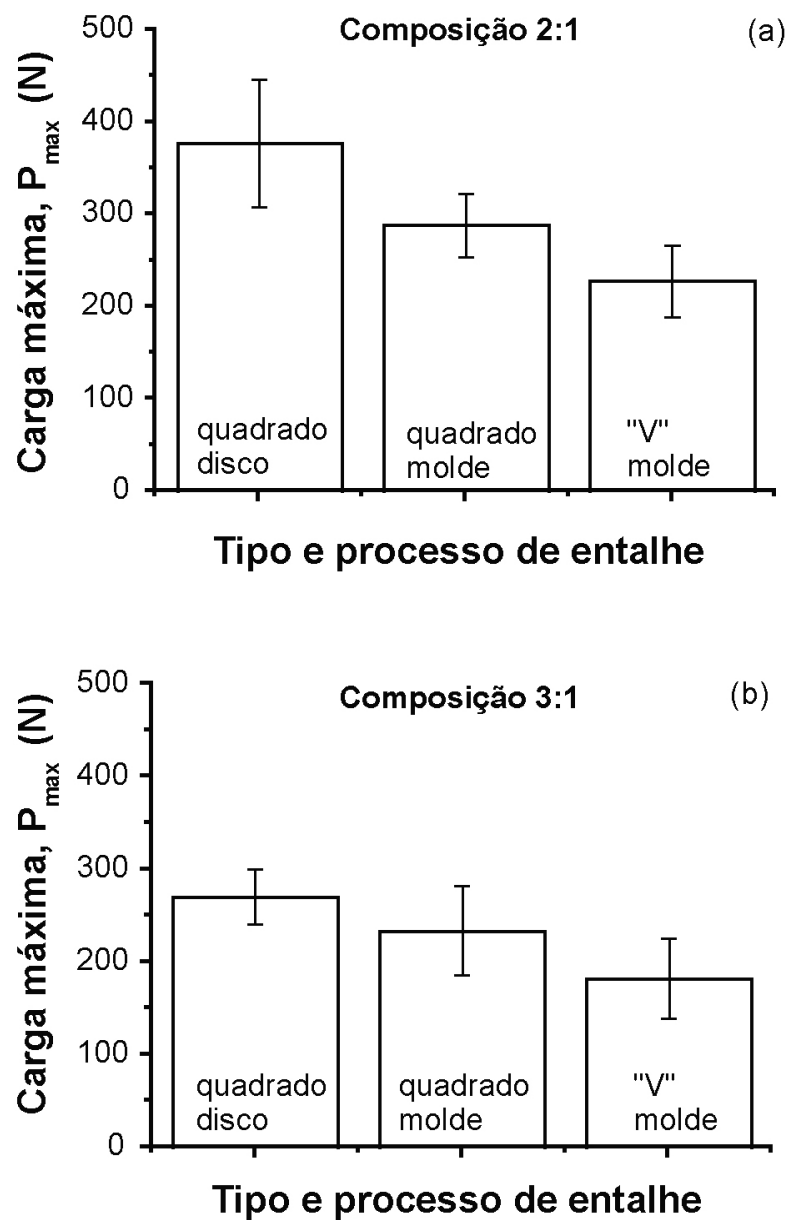

Figura 9: Influência da forma e do processo de obtenção do entalhe e da composição das argamassas na carga máxima: (a) composição 2:1 e (b) composição $3: 1$.

[Figure 9: Influence of the notch shape and preparation process and of the composition on the maximum load: (a) composition 2:1, and (b) composition 3:1.]

indicando que para esses materiais, é a altura geral da curva carga-deslocamento que está determinando o trabalho total de fratura, $\int \mathrm{P}_{\mathrm{M}} \mathrm{d} \delta$, mais do que o deslocamento do atuador para levar a propagação até o fim do corpo de prova. Como a forma do entalhe está afetando sensivelmente a carga máxima atingida durante a propagação, isso se reflete diretamente a energia de fratura. Talvez, se isso também afetasse o deslocamento total do atuador de algum modo sistemático (maior ou menor "flexibilidade" dos corpos de prova), o resultado poderia ser diferente, contrário ao encontrado ou reforçando o encontrado ou, ainda, compensando para manter $\gamma_{\text {wof }}$ independente dessa variável. Seria interessante encontrar microestruturas ou materiais que pudessem apresentar comportamentos distintos para se verificar as várias possibilidades.

\section{CONCLUSÕES}

As amostras para o método da cunha, visando determinar a energia de fratura de argamassas e concretos, podem ser

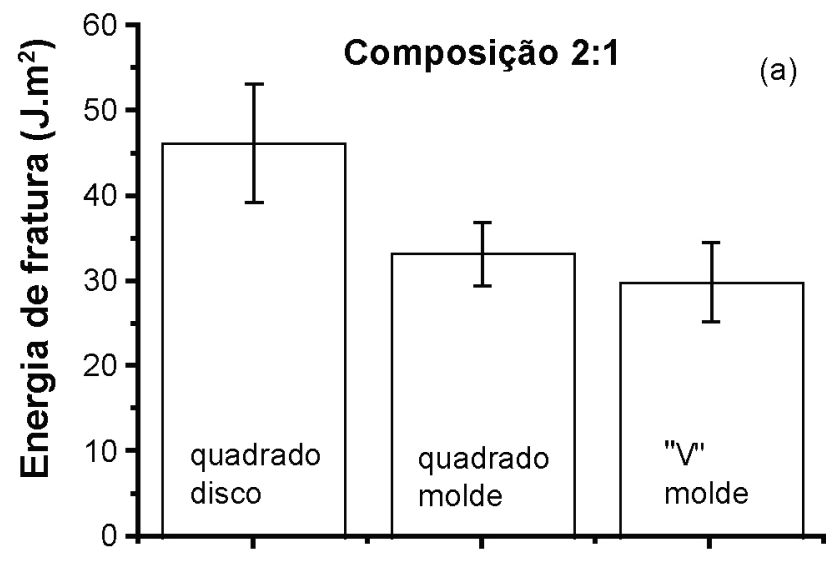

Tipo e processo de entalhe

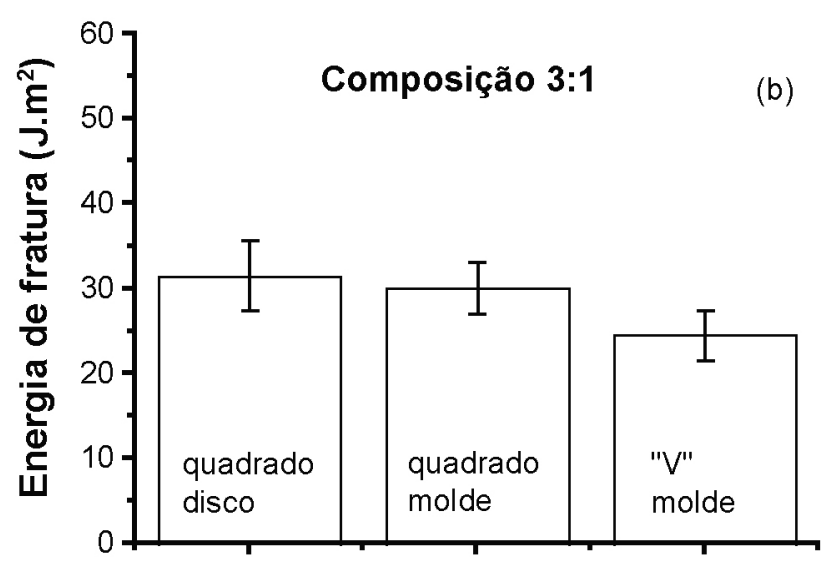

Tipo e processo de entalhe

Figura 10: Influência da forma e do processo de obtenção do entalhe e da composição das argamassas na energia de fratura: (a) composição 2:1 e (b) composição $3: 1$.

[Figure 10: Influence of the notch shape and preparation process and of the composition on the fracture energy: (a) composition 2:1, and $(b)$ composition $3: 1$.

produzidas já com entalhe e ranhuras durante a moldagem. $\mathrm{O}$ entalhe obtido na moldagem com ponta em forma de "V", além de mais barato que o produzido mecanicamente, pois este requer a utilização de disco diamantado além do custo de mão de obra e de equipamento, gera curvas carga-deslocamento perfeitamente estáveis, mesmo para argamassas mais resistentes mecanicamente, sob o ponto de vista de carga máxima atingida durante a propagação.

O efeito de parede é observado não só nas partes da amostras em contado com as paredes do molde e das lâminas como também nas superfícies dos agregados maiores, fazendo com que esses fiquem com uma camada de matriz bem distribuída ao longo de todo o volume da amostra.

Provavelmente a principal causa da carga máxima apresentada pelas amostras entalhadas com disco diamantado serem maiores que as entalhadas com o mesmo perfil, na própria moldagem, seja devido às diferenças de microestrutura nas pontas dos entalhes. A microestrutura das amostras entalhadas na moldagem é constituída 
principalmente pela parte fina da argamassa devido ao efeito de parede, enquanto as entalhadas com disco é formada pelos agregados presos pela matriz, daí necessitando de cargas mais elevadas para iniciar a formação e propagação das trincas, para ambas as composições estudadas. Porém, devese, também, considerar que o entalhe quadrado produzido pelo disco é aproximadamente o dobro mais largo do que aquele produzido pela lâmina do molde, sugerindo uma menor concentração de tensão em sua ponta e a necessidade de maior carga da máquina.

A composição da argamassa tem influência significativa tanto na carga máxima quanto na energia de fratura independentemente do tipo ou forma de entalhe.

As menores cargas máximas e conseqüentemente menores energias de fratura para as amostras entalhadas em "V", comparadas com as entalhadas com a forma quadrada, ambas com entalhes produzidos na moldagem, é devido à concentração de tensão na ponta do entalhe em "V", que é maior que no quadrado.

O perfil das curvas carga-deslocamento, na região da carga máxima, para as amostras de composição 3:1 é bem mais arredondado que para as amostras 2:1, mostrando que a estabilidade de propagação da trinca é bastante influenciada pela composição das argamassas e independentes do tipo de entalhe e forma de obtenção desses.

A região de interface agregado/matriz é mais fraca que os agregados, pois em todos os casos testados houve destacamento dos agregados e não a fratura dos mesmos.

\section{AGRADECIMENTOS}

À Fundação de Amparo à Pesquisa do Estado de São Paulo, FAPESP, Proc. 2001/04324-8, ao Conselho Nacional de Desenvolvimento Científico e Tecnológico, CNPq, pelas bolsas de Produtividade em Pesquisa, Procs. 304980/2003-0 e 301073/2006-6, pelo Projeto Universal, Proc. 470504/20048 e pela bolsa de Pós-Doutorado Sênior, Proc. 150038/20062. À Coordenação de Aperfeiçoamento de Pessoal de Nível Superior, CAPES, pelo projeto PROCAD, Proc. 0136050 e pelo projeto PROBRAL, Proc. 258/07.

\section{REFERÊNCIAS}

[1] A. Carpinteri, B. Chiara, K. M. Nemati, Mech. Mater. 29 (1997) 93.

[2] G. V. Guinea, K. E. Sayed, C. G. Rocco, M. Elices, J. Planas, Cem. Concr. Res. 32 (2002) 1961.

[3] B. L. Karihaloo, H. M. Abdalla, Q. Z. Xiao, Cem. Concr. Res. 36 (2006) 171.

[4] C. Rosselló, M. Elices, Cem. Concr. Res. 34 (2004) 1441.

[5] J. Xiao, H. Schneider, C. Dönnecke, G. König, Constr. Build. Mater. 18 (2004) 359.

[6] S. Erdogdu, S. Kurbetci, Cem. Concr. Compos. 27 (2005) 818.

[7] M. Bao-guo, W. Xin-gang, L. Wen-quan, L. Xiang-guo, H. Zhen, Constr. Build. Mater. 21 (2007) 2017.

[8] X. Xuan, S. Raghibizadeh, D. Li, J. Colloid Interface Sci.
296 (2006) 743.

[9] A. M. Vidales, I. Ippolito, Physica A 337 (2004) 495.

[10] A. Menou, G. Mounajed, H. Bousssa, A. Pineaud, H. Carre, Theor. App. Fract. Mech. 45 (2006) 64.

[11] K. R. Wu, B. Chen, W. Yao, D. Zhang, Cem. Concr. Res. 31 (2001) 1421.

[12] C-C. Chou, I-L. Cheng, K-J. Chen, Y-C. Ko, Am. Ceram. Soc. Bull. 65 (1986) 1042.

[13] Y-C. Ko, Ceram. Int. 27 (2001) 501.

[14] D. R. Larson, J. A. Coppola, D. P. H. Hasselman, J. Am. Ceram. Soc. 57 (1974) 417.

[15] H. Harmuth: Theor. App. Frac. Mech. 23 (1995) 103.

[16] J. Nakayama, H. Abe, R. C. Bradt, J. Eur. Ceram. Soc. 64 (1981) 671.

[17] S. Ribeiro, C. C. D. Exposito, J. A. Rodrigues, Cerâmica (2008) no prelo.

[18] H. Harmuth, K. Rieder, M. Krobath, E. Tschegg, Mater. Sci. Eng. A 214 (1996) 53.

[19] E. Tschegg, Republik Österreich, Patent n. 390328B registered 1986.

[20] R. Nilica, H. Harmuth, Cem. Concr. Res. 35 (2005) 1641.

[21] B. Alapin, M. Pllig, J. Pötschke, Proc. $46^{\text {th }}$ Int. Coll. Refract. Eurogress, Aachen, Alemanha (2003) 107.

[22] R. Walter, L. Ostergaard, J. F. Olesen, H. Stang, Eng. Fract. Mech. 72 (2005) 2565.

[23] H. Harmuth, K. Rieder, M. Krobath, E. Tschegg, Mater. Sci. Eng. A 214 (1996) 53.

[24] T. Auer, C. Manhart, H. Harmuth, RHI Bulletin 1 (2006) 38.

[25] J-K. Kim, Y. Lee, S.-T. Yi, Cem. Concr. Res. 34 (2004) 507.

[26] J-K. Kim, Y-Y. Kim, Cem. Concr. Res. 29 (1999) 705.

[27] S. Ribeiro, J. S. C. Vieira, C. C. D. Exposito, J. A. Rodrigues, Proc. 10 ${ }^{\text {th }}$ Unif. Int. Techn. Conf. Refract. (UNITECR), Dresden, Alemanha 1 (2007) 498.

[28] S. Ribeiro, J. S. C. Vieira, C. C. D. Exposito, J. A. Rodrigues, Anais do $51^{\circ}$ Cong. Bras. Cerâmica, Salvador, BA (2007) 1.

[29] D. M. Tan, E. K. Tschegg, H. Rotter, H. O. K. Kirchner, Acta Metall. Mater. 43 (1995) 3701.

[30] B. Trunk, G. Schober, A. K. Helbling, F. H. Wittmann, Cem. Concr. Res. 29 (1999) 855.

[31] L. Brian, Fracture of britle solids, $2^{\text {nd }}$ Ed., Cambridge University Press, Cambridge (1993) 378.

[32] R. Damani, R. Gstrein, R. Danzer, J. Eur. Ceram. Soc. 16 (1996) 695.

[33] T. Fett, J. Eur. Ceram. Soc. 25 (2005) 543.

[34] S. V. Kamat, J. P. Hirth, F. W. Zok, Acta Mater. 44 (1996) 1831.

[35] D. Picard, D. Leguillon, C. Putot, J. Eur. Ceram. Soc. 26 (2006) 1421.

[36] J. Y. Shen, J. P. Hirth, F. W. Zok, J. A. Heathcote, Scripta Mater. 38 (1998) 15.

[37] E. K. Tschegg, M. Elser, S. E. Stanzl-Tschegg, Cem. Concr. Compos. 17 (1995) 57.

[38] F. N. Cunha-Ducan, R. C. Bradt, Cerâmica 49 (2003) 199.

(Rec. 26/08/2008, Ac. 14/11/2008) 